\title{
Monocular Temporal Hemianopia due to Acute Zonal Occult Outer Retinopathy
}

\author{
Yusuf Ahmed $^{a}$ Aman Sayal $^{a} \quad$ Alexander J. Kaplan $^{\text {b, c }}$ \\ Jonathan A. Micielib, c \\ aFaculty of Medicine, University of Toronto, Toronto, ON, Canada; ${ }^{b}$ Department of \\ Ophthalmology and Vision Sciences, University of Toronto, Toronto, ON, Canada; \\ cKensington Vision and Research Centre, Toronto, ON, Canada
}

\section{Keywords}

Acute zonal occult outer retinopathy $\cdot$ Monocular temporal hemianopia $\cdot$ Retina

\begin{abstract}
Retinal disease may mimic an optic neuropathy since both may result in a relative afferent pupillary defect (RAPD), and retinal abnormalities may not be evident on a clinical exam. We report a case of a young woman with a monocular temporal hemianopia respecting the vertical meridian due to acute zonal occult outer retinopathy (AZOOR). This 34-year-old woman presented with a 10-day history of left eye vision loss and was found to have a visual acuity of 20/20 in both eyes, a left RAPD, and left temporal hemianopia on Humphrey 24-2 SITA-Fast visual field testing. Dilated fundus examination showed a normal-appearing optic nerve and retina in both eyes. She had already had a normal magnetic resonance imaging of the orbits with contrast and retinal disease was suspected. Optical coherence tomography showed dropout of the ellipsoid zone in the peripapillary retina, and fundus autofluorescence showed hyper-autoflourescence in the peripapillary region of the left eye. A diagnosis of AZOOR was made, and no improvement with prednisone occurred at final follow-up. This case demonstrates the importance of multimodal imaging in patients referred for optic neuropathies since retinal disease such as AZOOR can produce visual field defects characteristic of optic nerve disease.
\end{abstract}

\section{Karger $\stackrel{2}{\circ}$}




\section{Case Reports in Ophthalmology}

\section{Introduction}

A monocular temporal hemianopia has been well-recognized as a syndrome of the optic nerve [1]. Pituitary adenomas accounted for the most common cause of monocular temporal hemianopia in a case series of 24 patients, as compressive lesions can interrupt crossing nasal fibres at the optic chiasm [2]. Other causes in this case series included compression from craniopharyngiomas, tuberculum sella meningioma, and optic neuritis. Retinal pathology may mimic optic neuropathies and typically requires multimodal imaging such as optical coherence tomography (OCT) and fundus autofluorescence (FAF) for diagnosis. Here, we present a rare case of acute zonal occult outer retinopathy (AZOOR), mimicking an optic neuropathy with a visual field defect, monocular temporal hemianopia, that is usually seen with compressive para-chiasmal pathology.

\section{Case Presentation}

A 34-year-old woman of Indian origin presented with a 10-day history of left eye blurred vision. She also felt that her colour vision was reduced in that eye. There was no pain or pain with eye movements and no preceding viral prodrome. She had no known medical conditions and took no regular medications. She saw an ophthalmologist who documented a visual acuity of 20/20 in both eyes, a left relative afferent pupillary defect (RAPD), and temporal visual field defect on confrontation, and arranged for magnetic resonance imaging (MRI) of the orbits and brain with contrast that was reported as normal. She was then referred to neuro-ophthalmology for suspicion of optic neuritis.

Neuro-ophthalmic examination revealed a visual acuity of 20/20 OD and 20/20 OS, and there was a left RAPD; colour vision was 14/14 Ishihara colour plates in both eyes, and there was left red desaturation ( $80 \%$ of normal). Humphrey 24-2 SITA-Fast visual fields were normal in the right eye, and there was a temporal visual field defect that respected the vertical meridian in the left eye (Fig. 1). Dilated fundus examination was normal in both eyes apart from subtle peripapillary thickening in the left eye (Fig. 2a). Spectral-domain OCT (SD-OCT) of the retinal nerve fibre layer showed a normal average thickness in both eyes (102 $\mu \mathrm{m}$ OD and $103 \mu \mathrm{m}$ OS; Fig. 3a), and the ganglion cell layer-inner plexiform layer also showed a normal average thickness in both eyes ( $82 \mu \mathrm{m}$ OD and $80 \mu \mathrm{m}$ OS; Fig. 3b). The MRI orbits and brain were reviewed and showed no compressive lesion along the anterior visual pathways and no enhancement of the optic nerve. A primary retinal abnormality was therefore suspected. FAF was performed and showed well-demarcated hyper-autofluorescence around the optic nerve in the left eye (Fig. 2b). SD-OCT of the macula and optic nerve head were also completed and showed dropout of the ellipsoid zone in the peripapillary retina in the left eye (Fig. 3c). She underwent blood testing with quantiFERON gold, angiotensin-converting enzyme, and VDRL, which were all normal. Chest X-ray was also normal.

She was diagnosed with AZOOR and was started on a trial of prednisone $1 \mathrm{mg} / \mathrm{kg}$ for 1 month, but she did not appreciate any changes in her vision. The prednisone was tapered off. At follow-up 4 months later, her visual acuity was 20/20 in both eyes, and the visual field and examination were unchanged to the presentation.

\section{Discussion/Conclusion}

First reported in 1992 by Gass [3], AZOOR is a rare unilateral or bilateral syndrome of unknown aetiology causing visual field loss due to disruption of the outer retinal layers. It has a predilection for young Caucasian individuals and may be associated with preceding viral

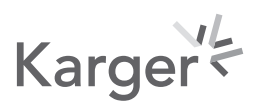




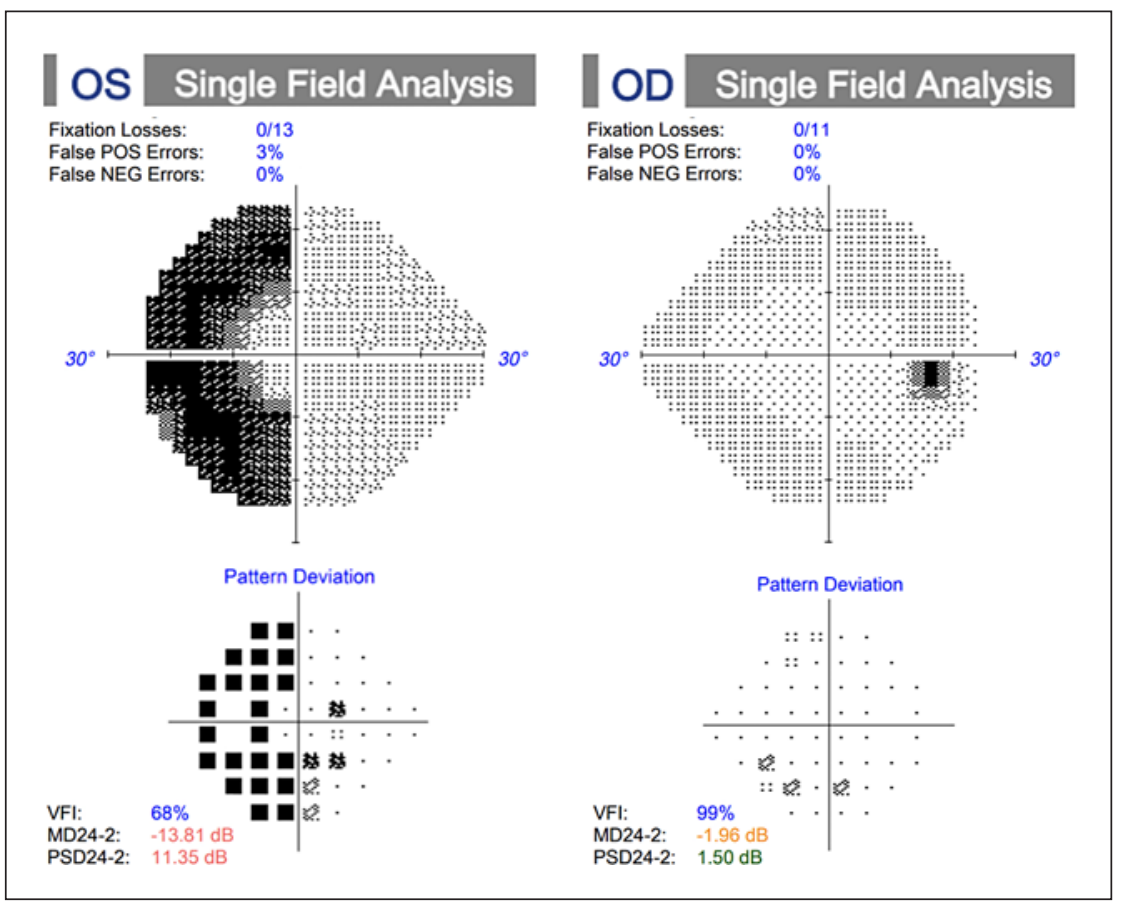

Fig. 1. Humphrey 24-2 SITA-Fast visual fields showing a left monocular temporal hemianopia.

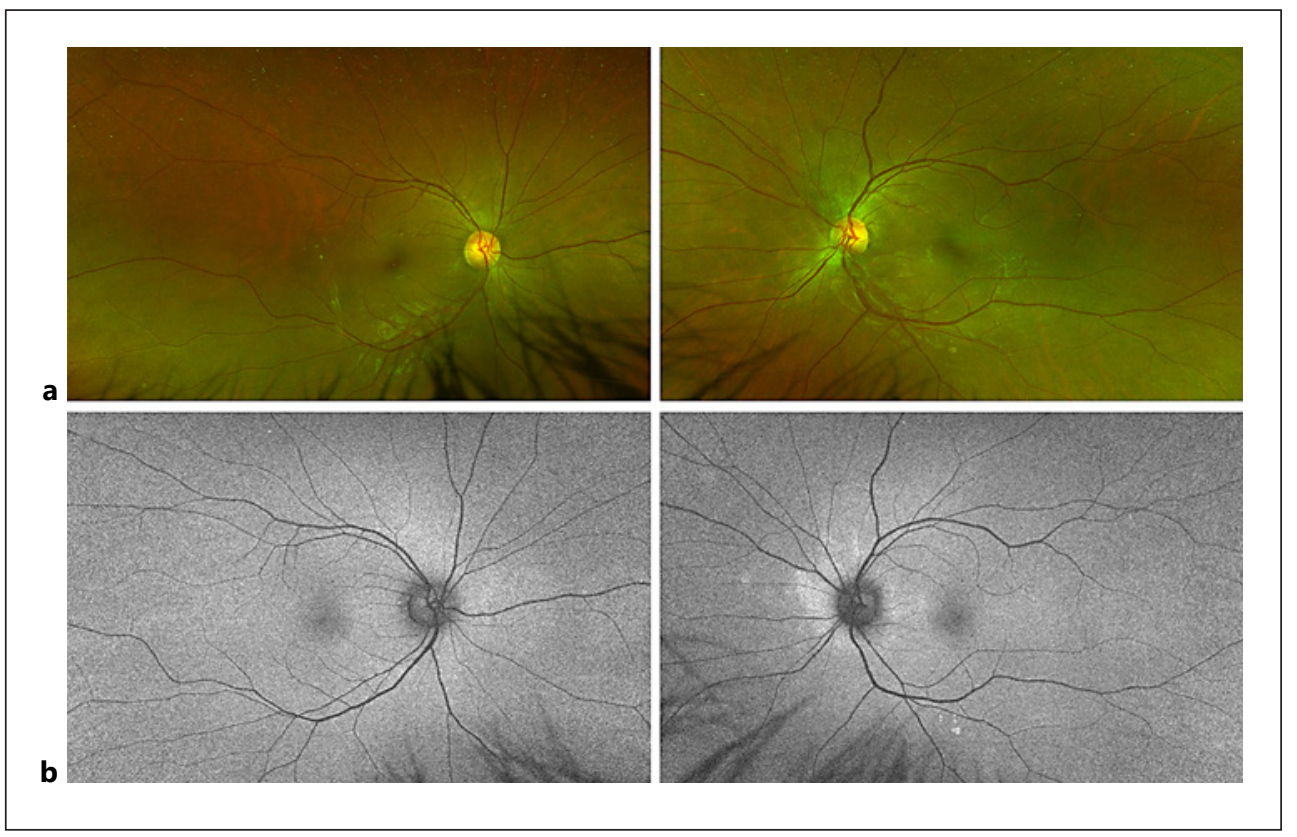

Fig. 2. Colour fundus photographs (a) demonstrating normal optic nerves in both eyes with mild peripapillary thickening in the left eye. FAF (b) demonstrating increased peripapillary FAF in the left eye.

illness [4]. Visual acuity is generally preserved in AZOOR, as retinal degeneration often spares the central fovea. Clinical examination typically reveals a normal fundus appearance [5]. Visual field testing is high yield as patients often demonstrate characteristic field loss patterns depending on the location of outer retinal disruption. AZOOR has been reported to most 


\section{Case Reports in Ophthalmology}

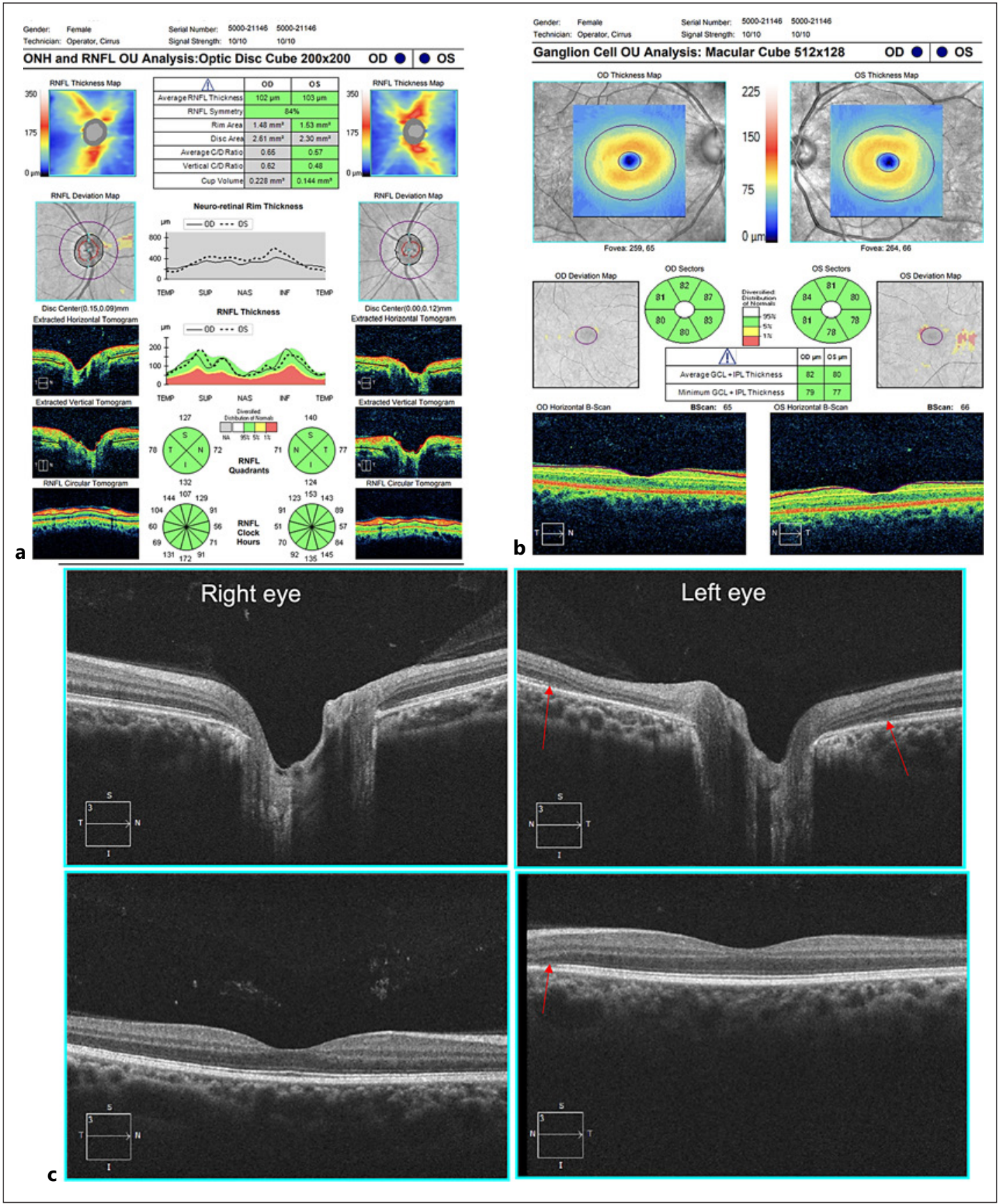

Fig. 3. OCT of the (a) retinal nerve fibre layer and (b) macular ganglion cell layer-inner plexiform layer showed normal average thicknesses. There is appreciable thickening in the left peripapillary area on the RNFL thickness map. c OCT of the peripapillary retina and macula showing dropout of the ellipsoid zone in the left eye (red arrows).

commonly cause peripapillary and nasal retinal damage - for this reason, enlarged blind spots or temporal field scotomas are most frequently observed [4].

Monocular temporal hemianopia is considered a visual field deficit produced by parachiasmal pathology [2]. Bitemporal hemianopia is also highly specific for compressive lesions to the optic chiasm [6] but has also been found to occur in AZOOR in rare circumstances $[7,8]$. 
In these cases, MRI or CT of the orbit and brain is ordered to rule out a sellar/suprasellar mass; however, there is diagnostic uncertainty when imaging does not reveal any abnormalities. Non-chiasmal aetiologies of monocular temporal hemianopia may include congenital opticdisc abnormalities [4], optic neuritis [2], sector retinitis pigmentosa [9], or be related to non-organic vision loss [10]. Amongst these organic causes, temporal visual field loss may occur if the retinal defect is confined to the nasal macula. To our knowledge, there are no reported cases of AZOOR causing monocular temporal hemianopia that respects the vertical meridian. As demonstrated in our patient, this possibility is conceivable if the outer retinal damage occurs in the nasal retina of one eye.

Multimodal ocular imaging with SD-OCT, FAF, indocyanine green angiography, and/or fluorescein angiography is useful in diagnosing AZOOR. Investigations will typically reveal a well-demarcated "transition zone" of retina appearing hyper-autofluorescent on FAF, as well as outer retinal disruption confined to the ellipsoid zone on SD-OCT. "Trizonal" involvement, referring to regions of healthy (zone 1), actively affected (zone 2), and atrophic (zone 3) retina, is also pathognomonic of AZOOR and may be observed on FAF and SD-OCT [5]. Electrophysiological abnormalities from electroretinography and electro-oculography testing can also be used to confirm diagnosis of AZOOR [4, 11].

It has been postulated that a genetic autoimmune/inflammatory reaction [12] or primary viral infection of the photoreceptors [13] may play a role in developing AZOOR; however, the pathogenesis of this syndrome is still unclear. Despite there being no wellestablished effective treatment for AZOOR, corticosteroids are most commonly used; in some cases, they have been shown to improve visual function [14]. Immunosuppressive and antiviral agents have also been trialled, albeit showing unclear clinical benefit $[15,16]$. Visual field deficits typically do not progress but are permanent beyond the acute stage of disease [4].

In summary, retinal pathology may mimic classic patterns of optic chiasm or optic neuropathies. Clinical examination is often not helpful in these situations since both will produce an RAPD. Adding OCT and FAF to the evaluation of patients with suspected optic neuropathies may reveal that the patient has a retinopathy and can reduce the need for costly MRIs. AZOOR is one such entity that can mimic optic chiasm pathology as demonstrated in this case.

\section{Statement of Ethics}

Written informed consent was obtained from the patients for publication of this case report and any accompanying images. This study protocol and retrospective review of patient data were reviewed, and the need for approval was waived by the University of Toronto Research Ethics Board.

\section{Conflict of Interest Statement}

None of the authors have any conflicts of interests or disclosures to declare.

\section{Funding Sources}

This research did not receive any specific grant from funding agencies in the public, commercial, or not-for-profit sectors.

\section{Karger'}




\section{Author Contributions}

Yusuf Ahmed, Aman Sayal, Dr. Alexander J. Kaplan, and Dr. Jonathan A. Micieli each contributed to the conception and design of this report, as well as drafting and revision of the manuscript. All the authors approved of this case report to be published.

\section{Data Availability Statement}

All data generated or analysed during this study are included in this article. Further enquiries can be directed to the corresponding author.

\section{References}

1 Lukewich MK, Micieli JA. Chronic chiasmal compression and persistent visual field defect without detectable changes in optical coherence tomography of the macular ganglion cell complex. Am J Ophthalmol Case Rep. 2019 Aug;16:100533.

2 Hershenfeld SA, Sharpe JA. Monocular temporal hemianopia. Br J Ophthalmol. 1993 Jul;77(7):424-7.

3 Gass JD. Acute zonal occult outer retinopathy. Donders Lecture: the Netherlands Ophthalmological Society, Maastricht, Holland, June 19, 1992. Retina. 1993 Jun;23(2):79-97.

4 Monson DM, Smith JR. Acute zonal occult outer retinopathy. Surv Ophthalmol. 2011 Jan;56(1):23-35.

5 Mrejen S, Khan S, Gallego-Pinazo R, Jampol LM, Yannuzzi LA. Acute zonal occult outer retinopathy: a classification based on multimodal imaging. JAMA Ophthalmol. 2014 Sep;132(9):1089-98.

6 Quencer RM. Visual loss and bitemporal hemianopsia. J Clin Neuroophthalmol. 1981 Sep;1(3):231-5.

7 Salgado CM, Dagnelie G, Miller NR. Bitemporal hemianopia caused by retinal disease. Arch Ophthalmol. 2009 Dec;127(12):1690-3.

8 Zaslavsky K, Eshtiaghi A, Jeeva-Patel T, Christakis PG, Margolin E. Bitemporal hemianopia secondary to acute zonal occult outer retinopathy. J Neuroophthalmol. 2021 Jan;41(4):e749-51.

9 Johnson LN, Rabinowitz YS, Hepler RS. Hemianopia respecting the vertical meridian and with foveal sparing from retinal degeneration. Neurology. 1989 Jun;39(6):872-3.

10 Gittinger JW Jr. Functional monocular temporal hemianopsia. Am J Ophthalmol. 1986 Feb;101(2):226-31.

11 Francis PJ, Marinescu A, Fitzke FW, Bird AC, Holder GE. Acute zonal occult outer retinopathy: towards a set of diagnostic criteria. Br J Ophthalmol. 2005 Jan;89(1):70-3.

12 Jampol LM, Becker KG. White spot syndromes of the retina: a hypothesis based on the common genetic hypothesis of autoimmune/inflammatory disease. Am J Ophthalmol. 2003 Mar;135(3):376-9.

13 Gass JD. Are acute zonal occult outer retinopathy and the white spot syndromes (AZOOR complex) specific autoimmune diseases? Am J Ophthalmol. 2003 Mar;135(3):380-1.

14 Chen SN, Yang CH, Yang CM. Systemic corticosteroids therapy in the management of acute zonal occult outer retinopathy. J Ophthalmol. 2015 Jan;2015:793026.

15 Mahajan VB, Stone EM. Patients with an acute zonal occult outer retinopathy-like illness rapidly improve with valacyclovir treatment. Am J Ophthalmol. 2010 Oct;150(4):511-8.

16 Spaide RF, Koizumi H, Freund KB. Photoreceptor outer segment abnormalities as a cause of blind spot enlargement in acute zonal occult outer retinopathy-complex diseases. Am J Ophthalmol. 2008 Jul;146(1): 111-20.

\section{Karger'k}

